\title{
Actualidad en estudios LibQUAL+@: paradigmas de la biblioteca informativa y social-creadora y cuestión de género como reflejos de la realidad social
}

\author{
Pedro Lázaro-Rodríguez*, Javier López-Gijón*, Sergio Alonso** y Enrique Herrera-Viedma*** \\ * Universidad de Granada. Facultad de Comunicación y Documentación \\ Correo-e: pedrolr@ugr.es | ORCID iD: https://orcid.org/0000-0002-8756-0507 \\ Correo-e: jgijon@ugr.es | ORCID iD: http://orcid.org/0000-0001-6517-8991 \\ ** Universidad de Granada. Departamento de Lenguajes y Sistemas Informáticos \\ Correo-e: zerjioi@ugr.es | ORCID iD: http://orcid.org/0000-0001-6620-328X \\ ** Universidad de Granada, Departamento de Ciencias de la Computación e Inteligencia Artificial \\ Correo-e: viedma@decsai.ugr.es | ORCID iD: http://orcid.org/0000-0002-7922-4984
}

Recibido: 12-02-2019; 2a versión: 10-06-2019; Aceptado: 26-06-2019

Cómo citar este artículo/Citation: Lázaro-Rodríguez, P.; López-Gijón, J.; Alonso, S.; Herrera-Viedma, E. (2020). Actualidad en estudios LibQUAL+\&: paradigmas de la biblioteca informativa y social-creadora y cuestión de género como reflejos de la realidad social. Revista Española de Documentación Científica, 43 (2), e264. https://doi.org/10.3989/redc.2020.2.1649

Resumen: Los objetivos de este artículo son cuatro: analizar las principales diferencias en torno a las dimensiones mejor valoradas de tres estudios sobre satisfacción de personas usuarias basados en LibQUAL+ $\mathbb{R}$ llevados a cabo en bibliotecas universitarias de España, Argentina y Chile; analizar las diferencias atendiendo a la variable de género (hombres y mujeres); determinar si las diferencias en cuanto a género son estadísticamente significativas; y discutir y reflexionar sobre los resultados hallados en el ámbito concreto de las bibliotecas como reflejo de la realidad social en general. Los resultados permiten concluir que en España las personas usuarias están menos satisfechas con la dimensión de la biblioteca como espacio, mientras que en Latinoamericana se percibe menor grado de satisfacción también con el control de la información. El mayor grado de satisfacción en todos los estudios se da en el valor afectivo del servicio. Las mujeres en España muestran menor satisfacción y mayor exigencia que los hombres en el valor afectivo del servicio. Además, se hallaron diferencias estadísticamente significativas en el análisis de las medias por género. En la discusión se ponen en relación los resultados hallados con la literatura existente, definiendo los paradigmas posibles de la biblioteca informativa frente al de la social-creadora y la percepción de la satisfacción por géneros según el contexto social en que se hallan las bibliotecas. La limitación principal del artículo es que se basa en tres estudios concretos; y su principal valor y originalidad, que puede considerarse como punto de partida para futuras investigaciones sobre la cuestión de género en lo que a bibliotecas y los servicios que ofrecen se refiere.

Palabras clave: Bibliotecas universitarias; LibQUAL+®; satisfacción; personas usuarias; género; evaluación de bibliotecas; biblioteca informativa; biblioteca social-creadora.

Current topics in LibQUAL+ $\circledast$ studies: the paradigms of the informative and the socialmaker library and the gender issue as reflections of the social reality

Abstract: The purposes of this article are four: to analyze the main differences between the best valued dimensions of three studies on users satisfaction based on LibQUAL+ ${ }^{\circledR}$ and carried out in university libraries of Spain, Argentina and Chile: to analyze the differences of the gender issue (men and women); to determine whether the differences on gender are statistically significant; and to discuss about the results in the specific field of libraries, as a reflection of the social reality in general. The results show that, in Spain, users are less satisfied with the dimension of the library as a space while in Latin America there is a lower degree of satisfaction about the control of information. The highest degree of satisfaction is found, in all the three studies, in the dimension related with the affective value of the service. Women in Spain show less satisfaction and a higher demand than men in the affective value of service. In addition, statistically significant differences were found in the analysis of the averages by gender. The results are discussed considering the reviewed literature and two paradigms are defined for libraries: the informative and the social-maker libraries, considering the satisfaction rates by gender as a reflection of the social reality. The main limitation of the article is that it is based on three specific studies. On the other hand, its main value and originality is that it can be considered as a starting point for further research on the gender issue in the field of libraries and their services.

Keywords: University libraries; LibQUAL $+\circledR$; satisfaction; library users; gender; library evaluation; informative library; social-maker library.

Copyright: (c) 2020 CSIC. Este es un artículo de acceso abierto distribuido bajo los términos de la licencia de uso y distribución Creative Commons Reconocimiento 4.0 Internacional (CC BY 4.0). 


\section{INTRODUCCIÓN}

La evaluación de bibliotecas puede entenderse como un ejercicio para conocer las principales carencias y debilidades de las mismas. También y más importante, como un ejercicio para conocer sus potencialidades entendidas como lo que pueden llegar a ser conociendo lo que son. La evaluación puede llevarse a cabo desde diferentes perspectivas, como son la objetiva y la subjetiva (Fushimi, 2011), siendo considerada como un medio para el conocimiento y la mejora de las bibliotecas (Gimeno-Perelló, 2008).

Tanto las evaluaciones objetivas como las subjetivas parten de datos que son procesados para obtener información. Dicha información puede obtenerse por medio del cálculo de una serie de indicadores cuyos resultados son interpretados para obtener una serie de conclusiones. En el primer sentido y focalizando el ámbito de las bibliotecas a las españolas, como ejemplo de evaluación objetiva cabe destacar el desarrollo y la publicación de la herramienta Secaba-Rank para conocer la eficiencia de las bibliotecas universitarias y las redes de lectura pública españolas (Lázaro-Rodríguez y otros, 2018a; Lázaro-Rodríguez y otros, 2019). Otra reciente investigación de este tipo trata de modelizar el uso de las bibliotecas públicas para los préstamos y las visitas a través de sus características en un estudio comparativo entre las de España y Finlandia (Lázaro-Rodríguez y Vakkari, 2018), hallando que el factor más fuerte para explicar tanto los préstamos como las visitas en España es la colección. Estos dos ejemplos parten de los datos en tanto que mediciones de la realidad ofrecidos por la web del Ministerio de Educación, Cultura y Deporte (MECD) de nombre Bibliotecas públicas españolas en cifras (MECD, 2020).

Pero también existen otras posibilidades para la toma de datos, como por ejemplo por medio de cuestionarios y encuestas. Cuando un cuestionario o encuesta se ofrece a personas usuarias de bibliotecas, puede decirse que la investigación toma un carácter subjetivo por cuanto los datos en los que se basará la investigación son dados por dichas personas usuarias. En ese sentido, si hay un modelo o estándar que sobresale por su aplicación sobre muchos otros en la evaluación de bibliotecas para la mejora de los servicios que ofrecen, ese es el llamado modelo o estándar LibQUAL $+\mathbb{B}$ (o derivados como LibQUAL+ @ Lite). Dicho estándar fue configurado a partir del modelo Servqual para la evaluación de servicios en general (Parasuraman y otros, 1988) y se entiende como un estudio de satisfacción de personas usuarias de bibliotecas para la evaluación y mejora de los servicios ofrecidos por las mismas.

La encuesta Libqual $+\AA$ consiste en una serie de ítems clasificados por lo general en tres dimensiones, a saber, la del valor afectivo del servicio, la biblioteca como espacio, y la del control de la información. Para cada ítem las personas usuarias dan valores comprendidos entre el 1 y el 9 para los niveles mínimo, observado y deseado. En base a dichos niveles, se calculan indicadores como el de la adecuación y la superioridad de los servicios. Estos indicadores ofrecen información acerca de la satisfacción de las personas usuarias con los servicios dados por las bibliotecas, y dicha información es el punto central y esencial que pueden considerar las bibliotecas para la mejora de sus servicios y la mayor satisfacción de las personas usuarias.

El grupo de investigación de los autores de este artículo ha llevado a cabo en los últimos años hasta siete estudios de satisfacción de personas usuarias en bibliotecas. Cuatro de ellos se basaron en la aplicación del modelo LibQUAL+ $®$. De estos cuatro estudios, tres se llevaron a cabo en bibliotecas universitarias de Argentina, Chile y España, y uno a una biblioteca pública española. En este trabajo se presentan y analizan los resultados de los estudios llevados a cabo en las bibliotecas universitarias por coincidir en tipología de biblioteca y considerando por eso mismo los resultados como comparables. La primera de las bibliotecas es la de la Universidad Católica de Argentina; la segunda, la de la Universidad Técnica Federico Santa María de Chile; y la tercera, la de la Universidad de Salamanca en España. De aquí en adelante se nombrarán como Argentina, Chile y Salamanca respectivamente.

La propuesta en este trabajo es, en primer lugar, analizar los resultados en relación a las dimensiones mejor valoradas en España y los países latinoamericanos. Para una hipótesis de partida al respecto se marca una basada en la común idea que asocia a las bibliotecas con la información, por lo que la hipótesis es que la dimensión mejor valorada será la del control de la información y no las de la biblioteca como espacio o el valor afectivo del servicio. En segundo lugar, y dado que en dos de los tres estudios se dispuso la posibilidad de clasificar los resultados por los géneros hombre y mujer, se pretende analizar las diferencias de los resultados de dichos dos estudios en base a la variable o cuestión de género. Para ello, en primer lugar se interpretarán los resultados atendiendo a las valoraciones para cada dimensión según la variable de género $y$, en segundo lugar, se llevará a cabo un análisis estadístico para comprobar si las diferencias en cada uno de los dos estudios son significativas. En cuanto a este segundo punto de la variable de género y en base a algunos estudios existentes (Hariri y Afnani, 2008; Cook y Thompson, 2001), como hipótesis de partida se marca la de que los resultados no serán significativamente diferentes.

Los objetivos de este trabajo pueden entenderse como la respuesta a las siguientes preguntas de investigación:

¿Cuáles son las principales diferencias en los resultados por dimensiones entre España y los países latinoamericanos considerando al total de personas usuarias? 
- ¿Cuáles son las principales diferencias en los resultados considerando la variable de género?

- ¿Son las diferencias en cuanto a la variable género estadísticamente significativas?

- ¿Son los resultados hallados para las tres cuestiones anteriores en el ámbito concreto de las bibliotecas reflejo de la realidad social?

En la siguiente sección se lleva a cabo una revisión de la literatura sobre dichos temas. A continuación, se expone todo lo concerniente a los materiales y metodología comprendiendo la sección metodológica y todo lo relacionado con la naturaleza de los estudios LibQUAL+ $®$ llevados a cabo y que son la base del presente trabajo. Tras ello, se exponen los resultados y su interpretación según las tres primeras preguntas de investigación definidas para mayor claridad. A ello sigue la discusión de los resultados en lo que equivale a la cuarta pregunta de investigación, relacionando también los resultados con los de los estudios incluidos en la revisión de la literatura. Por último, se dedica una sección para las conclusiones.

\section{REVISIÓN DE LA LITERATURA}

Los estudios de satisfacción de personas usuarias de bibliotecas basados en la metodología LibQUAL $+\circledR$ son numerosos a nivel internacional (LibQUAL $+\circledR$, 2020). El modelo LibQUAL+ $\AA$ fue implementado por Fred Heath, Collen Cook y más tarde Russell Thompson en el marco de la Association of Research Libraries (ARL) para su aplicación a bibliotecas como adaptación del modelo Servqual para evaluar la calidad de servicios en general (Parasuraman y otros, 1988). Por ello muchos de los primeros estudios LibQUAL+\& llevan la firma de Heath, Cook y Thompson y fueron llevados a cabo en bibliotecas de Estados Unidos y Canadá (Cook y otros, 2001; Cook, Heath y Thompson, 2001; Cook y Heath, 2001; Cook y Thompson, 2001).

Los estudios basados en la metodología LibQUAL+ $\&$ predominan sobre todo aplicados a bibliotecas universitarias y se encuentran casos de su aplicación en países como Irlanda (McCaffrey, 2013), Irán (Asemi y otros, 2010; Pourahmad y otros, 2016), Pakistán (Rehman, 2013), o Malasia (Dahan y otros, 2016). Para Europa, existen incluso trabajos que analizan el uso de LibQUAL $+\circledR$ por parte de bibliotecas en términos cuantitativos y en los que se deja constancia de su numerosa aplicación (Voorbij, 2012).

Otra línea de investigación en torno a Libqual toma por objeto de estudio al estándar mismo y no su aplicación en bibliotecas. Por ejemplo, existen estudios que analizan cómo el nivel de satisfacción en una dimensión o dimensiones puede afectar al nivel de las demás (Killick y otros, 2014); sobre si las personas usuarias son capaces de distinguir el sentido de los valores mínimo, observado y deseado (Natesan y Aerts, 2016); sobre evaluación de la fiabilidad, validez y estructura de versiones Libqual en un idioma distinto del original (Morales y otros, 2011); e incluso sobre si la inclusión de sorteos o premios en los estudios favorece la participación en los mismos (Buck y otros, 2012). También, en los últimos años se han propuesto nuevos modelos a partir del original Libqual basados en lógica difusa en trabajos que unen los campos de la inteligencia artificial con el de la biblioteconomía en particular y la documentación en general (Cabrerizo y otros, 2012; Cabrerizo y otros, 2017).

En el ámbito de España, aunque se encuentran algunos artículos aplicando la metodología Libqual a bibliotecas universitarias biomédicas (López-Gijón y otros, 2010), el número de estudios Libqual publicados como trabajos de investigación es más bien escaso. Una razón de ello puede ser extrapolada al tiempo actual desde la conclusión alcanzada por Rey-Martín (1999), y es que tal y como afirma la autora, muchos estudios de satisfacción de personas usuarias en bibliotecas son simplemente entregados a las bibliotecas estudiadas en vez de ser publicados como trabajos de investigación, algo que también comentó Delgado-López-Cózar (2004) en su trabajo sobre la investigación por encuesta en el campo de la biblioteconomía y la documentación en España.

Aunque las bibliotecas universitarias en que se basa el actual estudio lo son de universidades de diversa naturaleza (una técnica, otra católica y la última pública), y de países diferentes, el interés del trabajo radica precisamente en el análisis de los resultados y la reflexión a partir de los mismos sobre lo que está ocurriendo en bibliotecas universitarias de países de Europa como España y de Latinoamérica como Argentina y Chile. En ese sentido, el trabajo de comparación se basa en ideas propuestas por Peter Johan Lor en varios de sus trabajos sobre ciencias de la información, bibliotecas, biblioteconomía o documentación internacional y comparativa (Lor, 2008; Lor, 2017). Entre las motivaciones y razones que aúna el autor en el primer caso para llevar a cabo estudios comparativos e internacionales en la disciplina están los de la cooperación, la innovación, avanzar en conocimiento y la del entendimiento propio. Además, el autor concluye que para el desarrollo de una disciplina, en este caso la de estudios internacionales comparativos sobre bibliotecas, se han de superar las meras contribuciones anecdóticas y descriptivas en pos de las que sirven para explicar y comprender. Eso mismo puede entenderse como uno de los preceptos de este estudio.

A su vez, la comparación en biblioteconomía y documentación se legitima con otros estudios en que se analizan sistemas de bibliotecas tan dispares en sus características como los sistemas de bibliotecas públicas de países como Finlandia, Corea del Sur, Noruega, Países Bajos, Estados Unidos de América o España (Lázaro-Rodríguez y Vakkari, 2018; Oomes y otros, 2014; Sin y otros, 2016). 
Por último y en referencia a estudios Libqual en los que se analizan bibliotecas de diversas características, cabe resaltar publicaciones de la misma ARL en que se analizan bibliotecas universitarias de diversos países como Alemania, Reino Unido, Francia y Suecia (Cook y otros, 2014) y otras en que se incluyen bibliotecas universitarias de Estados Unidos y Canadá (ARL, 2018).

En cuanto a diferencias según la variable de género en bibliotecas en estudios de satisfacción de personas usuarias, se encuentran algunas aplicaciones tanto del modelo Servqual (Hossain-Shoeb, 2010; Hossain-Shoeb y Zabed-Ahmed, 2009) como del modelo Libqual (Hariri y Afnani, 2008). Aun con todo, los trabajos basados en Libqual que han reportado diferencias estadísticamente significativas entre las medias de hombres y mujeres para los indicadores de la adecuación y la superioridad por dimensiones y el total media de todas ellas predominan por su escasez (Hiller, 2001; Hariri y Afnani, 2008). Además, la idea de Cook y Thompson (2001) sobre que los resultados en Libqual no serán estadísticamente diferentes en cuanto a género, se ve avalada por otros autores en estudios concretos, como el llevado a cabo en la biblioteca de la Universidad de Washington, donde se encontraron diferencias significativas en encuestas propias pero no en las basadas en Libqual (Heinrichs y otros, 2005). En ese sentido, lo más reseñable y relevante de este último trabajo es la idea expresada por los autores acerca de que las diferencias de género representan una potencial área de investigación debido a que no se han podido establecer conclusiones firmes al respecto.

Con todo, la revisión de la literatura llevada a cabo permite extraer que, en general, abundan más los trabajos Libqual entregados a las bibliotecas estudiadas y no tanto los publicados como trabajos de investigación, especialmente en España. Además, la cuestión de la variable de género puede analizarse en mayor grado de tal forma que se puedan alcanzar conclusiones más sólidas en cuanto a la existencia o no de diferencias estadísticamente significativas entre las medias de ambos grupos. Desde ahí, se concibe como idóneo llevar a cabo un estudio como el que se propone en este trabajo tomando como base tres estudios LibQUAL $+\circledR$ en bibliotecas universitarias de tres países diferentes y con dos de ellos incluyendo el estudio de las diferencias en cuanto a la variable de género, dando respuesta y ampliando el conocimiento en torno a los temas y a las preguntas de investigación planteadas.

\section{MATERIALES Y MÉTODOS}

Este trabajo se basa en tres estudios de satisfacción de personas usuarias en bibliotecas que consisten en el estándar LibQUAL+ \& y comparten al menos los mismos ítems para las dimensiones del valor afectivo del servicio, la biblioteca como espacio y el control de la información.
Para cada ítem de la encuesta LibQUAL $+\circledR$ las personas usuarias dan tres valores, a saber, el mínimo (nivel por debajo del cual se piensa que el servicio no podría considerarse tal), el observado (nivel que se percibe u observa en la biblioteca durante el uso del servicio) y el deseado (nivel referido a lo que gustaría recibir idealmente del servicio). Estos datos que conforman las encuestas responden a una escala Likert del 1 (menor satisfacción) al 9 (mayor satisfacción). Desde ahí, los indicadores que se calculan son los de la adecuación (valor observado menos el mínimo) y la superioridad (valor observado menos el deseado). Con todo, una adecuación negativa indica que el valor observado es inferior al mínimo $y$, por tanto, que el servicio no se adecua al grado de satisfacción que las personas usuarias esperan como mínimo de los servicios. Por su lado, una superioridad negativa indica que el valor observado es menor que el deseado, esto es, que los servicios prestados no superan las expectativas de las personas usuarias. Con la adecuación y la superioridad positivas la situación sería la contraria, y fundamentaría la situación de calidad en las bibliotecas siendo el servicio ofrecido adecuado y/o superando las expectativas de las mismas.

En su análisis de la investigación por encuesta en la disciplina de la biblioteconomía y la documentación en España, Delgado-López-Cózar (2004) elabora una lista de control de 21 ítems en cuatro bloques sobre información mínima a incluir en el informe final de una encuesta para avalar su validez y fiabilidad. Además, recomienda pedir a las personas autoras de encuestas que incluyan en la sección metodológica del informe una ficha técnica donde se contemplen los 21 ítems ofrecidos en su trabajo. Los cuatro bloques se refieren a información sobre la población, la muestra, la metodología de las encuestas, e información sobre el tratamiento de los datos. En aras a cumplir con las recomendaciones del autor se han diseñado cuatro tablas en las que se muestra la información clasificada según los bloques mencionados para los tres estudios en que se basa este trabajo.

La información sobre la población de los tres estudios se muestra en la Tabla I.

Sobre la Tabla I cabe especificar la dificultad de definir el tamaño de la población en todos los casos menos en Chile al incluirse, por ejemplo, grupos de personas usuarias como "usuario externo" u "otros" en los casos de Argentina y Salamanca. Cabe señalar que los estudios de satisfacción de usuarios tipo Libqual se realizan esencialmente sobre personas usuarias reales de la biblioteca, que son las que hacen uso de los servicios y pueden valorar lo cuestionado. En ese sentido, hay que considerar la diferenciación entre las personas usuarias reales y las potenciales, que serían en cambio las que no han hecho uso de los servicios de la biblioteca. Al respecto y como tema actual de interés, cabe resaltar la existencia de estudios sobre biblio- 
tecas que consideran la valoración de las mismas por parte de las personas no usuarias en mismo grado de importancia que las usuarias para su mejora (Fernández-Ardèvol y otros, 2018).

La información sobre la muestra de los tres estudios se incluye en la Tabla II.

Por su lado, la información sobre la metodología de los tres estudios se muestra en la Tabla III.

En referencia al ítem sobre el tipo de cuestionario de la Tabla III en los tres casos se recoge información para al menos las dimensiones del valor afectivo, la biblioteca como espacio y el control de la información, y que cada estudio añadió alguna pre- gunta adicional del tipo "observaciones generales" o "frecuencia de asistencia a la biblioteca". En cuanto a la inserción de los cuestionarios, se han añadido para su consulta desde un repositorio abierto de datos $^{1}$. Por otro lado, cabe señalar que las fechas de realización de las encuestas coinciden con los periodos próximos a las evaluaciones finales en los casos de Salamanca y Chile y con los de la primera evaluación de junio y julio en el caso de Argentina. En ese sentido, se trata de periodos donde el uso de las bibliotecas es mayor y enfocado al estudio por la proximidad a las evaluaciones.

Por último, la información sobre el tratamiento de los datos de los tres estudios se muestra en la Tabla IV.

Tabla I. Información sobre la población

\begin{tabular}{|l|c|c|c|}
\hline \multicolumn{1}{|c|}{ Ítem } & Argentina & Chile & Salamanca \\
\hline $\begin{array}{l}\text { 1. Identificación clara y } \\
\text { precisa de la población } \\
\text { (personas usuarias por } \\
\text { grupos) }\end{array}$ & $\begin{array}{c}\text { Autoridad, administrativo, } \\
\text { estudiantes de grado, } \\
\text { de posgrado, graduado, } \\
\text { investigador, profesor, } \\
\text { usuario externo }\end{array}$ & $\begin{array}{c}\text { PDI, estudiante de } \\
\text { pregrado, estudiante de } \\
\text { postgrado, PAS }\end{array}$ & $\begin{array}{c}\text { Estudiantes de grado, } \\
\text { de posgrado (máster y } \\
\text { doctorado), PDI, PAS, otros }\end{array}$ \\
\hline $\begin{array}{l}\text { 2. Descripción de la lista } \\
\text { usada para identificar la } \\
\text { población }\end{array}$ & \multicolumn{2}{|c|}{ Datos de cada universidad } \\
\hline 3. Tamaño de la población & No ofrecido desde la Universidad/biblioteca & 30989 \\
\hline
\end{tabular}

Nota: PDI equivale a Personal Docente e Investigador y PAS a Personal de Administración y Servicios. El tamaño de la población en el caso de Salamanca se ha obtenido del Observatorio de la Calidad y el Rendimiento Académico de la Universidad de Salamanca en el curso académico 2016-2017 (Universidad de Salamanca, 2020). Dicha cifra no incluye las personas usuarias del grupo "otros".

Tabla II. Información sobre la muestra

\begin{tabular}{|c|c|c|c|}
\hline Ítem & Argentina & Chile & Salamanca \\
\hline $\begin{array}{l}\text { 4. Identificación clara y } \\
\text { precisa de la muestra }\end{array}$ & \multirow{2}{*}{\multicolumn{3}{|c|}{$\begin{array}{l}\text { Personas que quieren contestar y participar en la encuesta por iniciativa propia o vol- } \\
\text { untaria. Por ello se entiende que se trata de personas usuarias reales de las bibliotecas } \\
\text { que han usado los servicios y pueden valorarlos. }\end{array}$}} \\
\hline $\begin{array}{l}\text { 5. Procedimiento de } \\
\text { muestreo }\end{array}$ & & & \\
\hline $\begin{array}{l}\text { 6. Tamaño de la muestra } \\
\text { (total de encuestas } \\
\text { recogidas) }\end{array}$ & 832 & 1726 & 1404 \\
\hline $\begin{array}{l}\text { 7. Cálculo tamaño de la } \\
\text { muestra }\end{array}$ & \multirow{3}{*}{\multicolumn{3}{|c|}{ 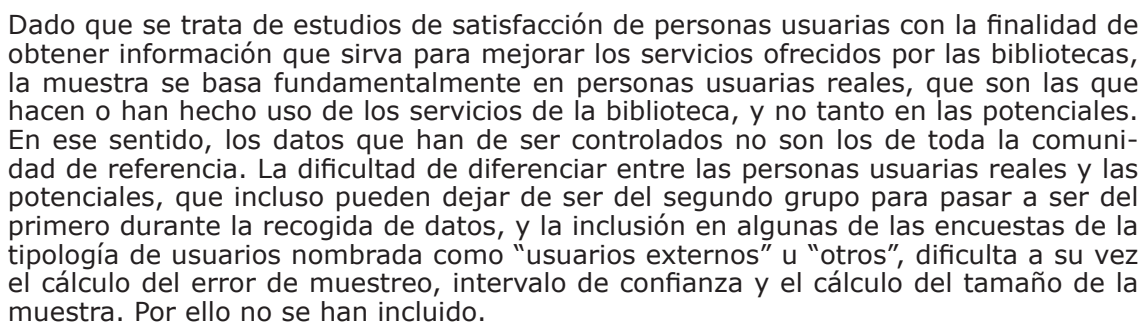 }} \\
\hline 8. Error de muestreo & & & \\
\hline 9. Intervalo de confianza & & & \\
\hline
\end{tabular}


Tabla III. Información sobre metodología de la encuesta

\begin{tabular}{|c|c|c|c|}
\hline Ítem & Argentina & Chile & Salamanca \\
\hline $\begin{array}{l}\text { 10. Fechas de realización } \\
\text { de la encuesta }\end{array}$ & 22/05/2018-13/07/2018 & $10 / 10 / 2017-27 / 12 / 2017$ & $2 / 05 / 2017-31 / 05 / 2017$ \\
\hline $\begin{array}{l}\text { 11. Modalidad de la } \\
\text { encuesta }\end{array}$ & \multicolumn{3}{|c|}{ Cuestionario en línea } \\
\hline 12. Tipo de cuestionario & \multicolumn{3}{|c|}{ Estándar LibQUAL+@ } \\
\hline $\begin{array}{l}\text { 13. Inserción del } \\
\text { cuestionario }\end{array}$ & \multicolumn{3}{|c|}{$\begin{array}{c}\text { Se añaden enlaces para el cuestionario de Argentina, Chile y Salamanca (ver nota } 1 \text { del } \\
\text { artículo) }\end{array}$} \\
\hline $\begin{array}{l}\text { 14. Prueba del } \\
\text { cuestionario }\end{array}$ & \multicolumn{3}{|c|}{ Todos los casos fueron probados por cada biblioteca antes de abrirse al público general } \\
\hline $\begin{array}{l}\text { 15. Información sobre los } \\
\text { encuestadores }\end{array}$ & \multicolumn{3}{|c|}{$\begin{array}{l}\text { Este ítem alude a encuestas realizadas por medio de entrevista, por lo que se obvia lo } \\
\text { concerniente al mismo }\end{array}$} \\
\hline $\begin{array}{l}\text { 16. Métodos de promoción } \\
\text { de la participación }\end{array}$ & \multicolumn{3}{|c|}{$\begin{array}{l}\text { Cada biblioteca se encargó de la promoción mediante carteles impresos, vía correo } \\
\text { electrónico y anuncios en las páginas web respectivas de las bibliotecas }\end{array}$} \\
\hline $\begin{array}{l}\text { 17. incidentes en el } \\
\text { desarrollo de la encuesta }\end{array}$ & $\begin{array}{c}\text { Por petición de la biblioteca, } \\
\text { se añadió una explicación } \\
\text { más extensa con ejemplos } \\
\text { sobre lo que son el valor } \\
\text { mínimo, observado y de- } \\
\text { seado }\end{array}$ & $\begin{array}{c}\text { Por petición de la bibliote- } \\
\text { ca, se modificó el requisito } \\
\text { de tener que contestar a } \\
\text { todas las preguntas de la } \\
\text { encuesta }\end{array}$ & Sin incidentes \\
\hline
\end{tabular}

Tabla IV. Información sobre el tratamiento de los datos

\begin{tabular}{|c|c|c|c|}
\hline Ítem & Argentina & Chile & Salamanca \\
\hline $\begin{array}{l}\text { 18. Tasa general de } \\
\text { respuesta }\end{array}$ & $\begin{array}{l}\text { - } 832 \text { encuestas recogidas } \\
\text { - } 733 \text { encuestas válidas } \\
\text { - } 732 \text { con todos los ítems } \\
\text { contestados ( } 276 \text { por } \\
\text { hombres y } 456 \text { por mu- } \\
\text { jeres) } \\
\text { - } 87,98 \% \text { de encuestas vál- } \\
\text { idas con todos los ítems } \\
\text { contestados respecto del } \\
\text { total de recogidas. }\end{array}$ & $\begin{array}{l}\text { - } 1726 \text { encuestas recogidas } \\
\text { - } 1723 \text { encuestas válidas } \\
\text { - } 630 \text { con todos los ítems } \\
\text { contestados } \\
\text { - } 36,50 \% \text { de encuestas vál- } \\
\text { idas con todos los ítems } \\
\text { contestados respecto del } \\
\text { total de recogidas. }\end{array}$ & $\begin{array}{l}\text { - } 1404 \text { encuestas recogidas } \\
\text { - } 1225 \text { encuestas válidas } \\
\text { - } 1225 \text { con todos los ítems } \\
\text { contestados ( } 480 \text { por } \\
\text { hombres y } 745 \text { por mu- } \\
\text { jeres) } \\
\text { - } 87,25 \% \text { de encuestas vál- } \\
\text { idas con todos los ítems } \\
\text { contestados respecto del } \\
\text { total de recogidas. }\end{array}$ \\
\hline $\begin{array}{l}\text { 19. Tasa respuesta } \\
\text { ítems (considerando las } \\
\text { encuestas válidas) }\end{array}$ & $\begin{array}{l}1 \text { encuesta con algún ítem } \\
\text { sin contestar }(99,86 \% \text { con } \\
\text { todos los ítems contestados } \\
\text { respecto de las válidas) }\end{array}$ & $\begin{array}{l}1093 \text { encuestas con algún } \\
\text { ítem sin contestar }(36,56 \% \\
\text { con todos los ítems } \\
\text { contestados respecto de las } \\
\text { válidas) }\end{array}$ & $\begin{array}{l}\text { Todas las encuestas con } \\
\text { todos los ítems contestados } \\
\text { (100\% con todos los ítems } \\
\text { contestados respecto de las } \\
\text { válidas) }\end{array}$ \\
\hline $\begin{array}{l}\text { 20. Programa empleado } \\
\text { en el tratamiento de los } \\
\text { datos }\end{array}$ & \multicolumn{3}{|c|}{$\begin{array}{l}\text { Los datos o resultados de las encuestas se recogieron y guardaron en una base de datos } \\
\text { mysql que fue exportada a formato csv para su tratamiento y procesamiento (cálculo de los } \\
\text { indicadores de la adecuación y la superioridad) en la hoja de cálculo LibreOffice Calc versión } \\
\text { 6.0.5.2. De ahí, se exportaron los datos de nuevo en formato csv para el análisis estadístico } \\
\text { sobre la variable de género en el software IBM SPSS } 24.0 \text { Desktop Linux Client Multilingual. }\end{array}$} \\
\hline $\begin{array}{l}\text { 21. Técnica empleada en } \\
\text { el análisis de datos }\end{array}$ & \multicolumn{3}{|c|}{$\begin{array}{l}\text { Desde los datos se calcularon los indicadores de la adecuación y la superioridad por dimen- } \\
\text { siones y para el total media de las tres dimensiones. Para el análisis estadístico de los resul- } \\
\text { tados en cuanto a la variable de género se estudió la normalidad de las variables mediante la } \\
\text { prueba de Kolmogorov-Smirnov. Dado que no se halló normalidad en ningún caso, se utilizó } \\
\text { la prueba no paramétrica U de Mann-Whitney aplicada a dos muestras independientes. }\end{array}$} \\
\hline
\end{tabular}


En cuanto al ítem sobre la tasa general de respuesta de la Tabla IV cabe especificar que, dado que en algunos casos no se conoce el tamaño de la población o muestra, o es indefinido por la existencia de grupos del tipo "usuarios externos" u "otros", se presenta información sobre encuestas recogidas, encuestas válidas, encuestas con todos los ítems contestados especificando las dadas por hombres y mujeres, y la tasa de las válidas con todos los ítems contestados respecto del total recogidas. A su vez, el proceso de análisis para definir el conjunto de encuestas válidas tras el total de recogidas consistió en todos los casos en la definición de una serie de filtros sobre encuestas que tardaron más del doble del tiempo medio en contestarse, encuestas que tardaron menos de 5 minutos en contestarse, encuestas con preguntas con el valor mínimo mayor que el deseado, y un último filtro para posibles encuestas contestadas repetidas (con todos los valores iguales). Estos conjuntos de encuestas se clasificaron en listas gracias a los filtros definidos y se analizaron manualmente para decidir su validez y su inclusión en el total de encuestas válidas para cada estudio.

Aunque la parte propiamente de metodología de los estudios se ha incluido en los ítems 20 y 21 de la Tabla IV, cabe resaltar que para el estudio de las diferencias estadísticamente significativas en cuanto al género en los estudios de Argentina y Salamanca se utilizó la prueba no paramétrica U de Mann-Whitney tras analizar la normalidad de las variables mediante la prueba de Kolmogorov-Smirnov y no hallarse normalidad.

Al respecto, existen estudios científicos que analizan las diferencias estadísticamente significativas de grupos en torno a la satisfacción de personas usuarias que utilizan la prueba $\mathrm{T}$ de student para 2 muestras independientes (Hariri y Afnani, 2008), y otros que utilizan la prueba no paramétrica $U$ de Mann-Whitney (Maidul-Islam y Zabed-Ahmed, 2011; Collins y Sto- ne, 2014; Yesmin y Zabed-Ahmed, 2016; Ahmed, 2017). Estos trabajos sirven de base y ejemplo para el presente estudio y la metodología empleada.

Por último, se determinó la consistencia interna de las encuestas de los tres estudios una vez procesadas las respuestas y considerando las encuestas válidas con todos los ítems contestados. El coeficiente alfa de Cronbach ha sido aplicado en diversos estudios para determinar la fiabilidad del estándar Libqual, derivados y adaptaciones a otros idiomas (Dahan y otros, 2016; Thompson y Cook, 2002; Thompson y otros, 2005; Toledo-Sánchez y otros, 2012). Los resultados del coeficiente alfa de Cronbach se muestran en la Tabla V.

Como se puede ver, para las tres encuestas que conforman este estudio los resultados del alfa de Cronbach por niveles del mínimo, observado y deseado, el total por dimensiones y el total de cada encuesta oscilan entre 0,811 y 0,969 . Dado que en el terreno de las ciencias sociales se recomienda que el coeficiente sea mayor que 0,7 (Nunnally, 1978) e igualmente en estudios sobre la fiabilidad de Libqual $+\circledR$ en su versión española (Morales y otros, 2011), la consistencia interna y la fiabilidad de las tres encuestas queda probada.

\section{RESULTADOS}

Desde los resultados para cada encuesta por ítem de cada biblioteca², y en base a otros trabajos científicos (Heinrichs y otros, 2005; Hariri y Afnani, 2008; Asemi y otros, 2010; Hossain-Shoeb, 2010; López-Gijón y otros, 2010; Cook y otros 2014; Killick y otros, 2014; ARL, 2018), se calcularon los resultados media de los valores mínimos, observados, deseados, y de los indicadores de la adecuación y la superioridad de los tres estudios por dimensiones y del total media de las tres dimensiones. Estos últi-

Tabla V. Resultados del coeficiente alfa de Cronbach

\begin{tabular}{|c|c|c|c|c|}
\hline Dimensión & Medida & Salamanca & Chile & Argentina \\
\hline \multirow{4}{*}{ Valor Afectivo (9 ítems) } & Mínimos & 0,965 & 0,959 & 0,969 \\
\hline & Observados & 0,959 & 0,940 & 0,948 \\
\hline & Deseados & 0,914 & 0,945 & 0,941 \\
\hline & Total dimensión & 0,949 & 0,949 & 0,948 \\
\hline \multirow{4}{*}{ Biblioteca como espacio ( 5 ítems) } & Mínimos & 0,920 & 0,925 & 0,944 \\
\hline & Observados & 0,851 & 0,887 & 0,874 \\
\hline & Deseados & 0,811 & 0,933 & 0,883 \\
\hline & Total dimensión & 0,873 & 0,902 & 0,892 \\
\hline \multirow{4}{*}{ Control de la información (8 ítems) } & Mínimos & 0,956 & 0,940 & 0,952 \\
\hline & Observados & 0,905 & 0,904 & 0,913 \\
\hline & Deseados & 0,892 & 0,917 & 0,926 \\
\hline & Total dimensión & 0,927 & 0,934 & 0,930 \\
\hline \multicolumn{2}{|l|}{ Total estudios } & 0,964 & 0,964 & 0,965 \\
\hline
\end{tabular}


mos se muestran en la Tabla VI para el total y por géneros en los casos que incluían dicho filtro.

De aquí en adelante y para una mayor claridad en la exposición e interpretación de los resultados, estos se presentan en tres puntos diferenciados siempre a partir de la Tabla VI: el primero se refiere a las diferencias principales por dimensiones entre España y los países latinoamericanos atendiendo al total (sin variable de género); el segundo, a las principales diferencias en los resultados considerando la variable de género en los estudios que la incluyen; y el último al análisis estadístico sobre las diferencias por géneros.

\subsection{Diferencias principales por dimensiones entre España y los países latinoamericanos considerando al total de personas usuarias}

En cuanto al mínimo, en el caso de Salamanca la dimensión con un mínimo más alto es la del valor afectivo del servicio $(6,02)$, frente a la del espacio $(5,94)$ y a la del control de la información $(5,91)$. Por su lado, en Chile el mínimo más alto se da en el valor afectivo del servicio $(6,02)$, frente al espacio $(6,00)$ y el control de la información $(5,71)$. Finalmente, en Argentina el mínimo más alto se da en el espacio $(6,74)$, frente al valor afectivo $(6,60)$ y el control de la información $(6,24)$. Por tanto, contando con que el mínimo se refiere al valor por debajo del cual se piensa que el servicio no podría considerarse tal, y punto a partir del cual el servicio se consideraría propiamente un servicio, en dos bibliotecas (Salamanca y Chile) el mínimo más alto se da en el valor afectivo del servicio y en la de Argentina se da en la dimensión del espacio. Es decir, en todas las bibliotecas se exige más valor afectivo del servicio o espacio que control de la información.

Atendiendo a los resultados para el valor observado, en Salamanca la dimensión con el resultado más alto es la del valor afectivo con 7,22. Por su lado, el valor observado más alto en el caso de

Tabla VI. Resultados de los valores e indicadores

\begin{tabular}{|c|c|c|c|c|c|c|c|c|c|c|c|c|c|c|c|}
\hline \multirow{3}{*}{ Biblioteca } & \multicolumn{15}{|c|}{ Valor afectivo del servicio } \\
\hline & \multicolumn{3}{|c|}{ Mín } & \multicolumn{3}{|c|}{ Obs } & \multicolumn{3}{|c|}{ Des } & \multicolumn{3}{|c|}{ Ade } & \multicolumn{3}{|c|}{ Sup } \\
\hline & $\mathbf{T}$ & $\mathbf{H}$ & M & $\mathbf{T}$ & $\mathbf{H}$ & M & $\mathbf{T}$ & $\mathbf{H}$ & M & $\mathbf{T}$ & $\mathbf{H}$ & M & $\mathbf{T}$ & H & M \\
\hline Salamanca & 6,02 & 5,99 & 6,04 & 7,22 & 7,24 & 7,20 & 8,42 & 8,39 & 8,45 & 1,20 & 1,26 & 1,16 & $-1,21$ & $-1,14$ & $-1,25$ \\
\hline Argentina & 6,60 & 6,51 & 6,65 & 7,78 & 7,66 & 7,86 & 8,47 & 8,33 & 8,55 & 1,18 & 1,15 & 1,20 & $-0,69$ & $-0,67$ & $-0,70$ \\
\hline Chile & 6,02 & - & - & 7,11 & - & - & 8,01 & - & - & 1,09 & - & - & $-0,90$ & - & - \\
\hline \multirow{3}{*}{ Biblioteca } & \multicolumn{15}{|c|}{ Biblioteca como espacio } \\
\hline & \multicolumn{3}{|c|}{ Mín } & \multicolumn{3}{|c|}{ Obs } & \multicolumn{3}{|c|}{ Des } & \multicolumn{3}{|c|}{ Ade } & \multicolumn{3}{|c|}{ Sup } \\
\hline & $\mathbf{T}$ & $\mathbf{H}$ & M & $\mathbf{T}$ & $\mathbf{H}$ & M & $\mathbf{T}$ & $\mathbf{H}$ & M & $\mathbf{T}$ & $\mathbf{H}$ & M & $\mathbf{T}$ & H & M \\
\hline Salamanca & 5,94 & 5,84 & 6,00 & 6,56 & 6,45 & 6,64 & 8,44 & 8,35 & 8,50 & 0,63 & 0,61 & 0,64 & $-1,88$ & $-1,91$ & $-1,86$ \\
\hline Argentina & 6,74 & 6,75 & 6,74 & 7,83 & 7,77 & 7,87 & 8,57 & 8,48 & 8,63 & 1,09 & 1,02 & 1,13 & $-0,74$ & $-0,71$ & $-0,76$ \\
\hline Chile & 6,00 & - & - & 6,31 & - & - & 8,13 & - & - & 0,31 & - & - & $-1,82$ & - & - \\
\hline \multirow{3}{*}{ Biblioteca } & \multicolumn{15}{|c|}{ Control de la información } \\
\hline & \multicolumn{3}{|c|}{ Mín } & \multicolumn{3}{|c|}{ Obs } & \multicolumn{3}{|c|}{ Des } & \multicolumn{3}{|c|}{ Ade } & \multicolumn{3}{|c|}{ Sup } \\
\hline & $\mathbf{T}$ & H & M & $\mathbf{T}$ & $\mathbf{H}$ & M & $\mathbf{T}$ & H & M & $\mathbf{T}$ & H & M & $\mathbf{T}$ & H & M \\
\hline Salamanca & 5,91 & 5,85 & 5,94 & 6,69 & 6,62 & 6,73 & 8,35 & 8,32 & 8,37 & 0,78 & 0,76 & 0,79 & $-1,66$ & $-1,71$ & $-1,63$ \\
\hline Argentina & 6,24 & 6,244 & 6,243 & 6,93 & 6,89 & 6,96 & 8,31 & 8,15 & 8,40 & 0,69 & 0,64 & 0,72 & $-1,37$ & $-1,27$ & $-1,44$ \\
\hline Chile & 5,71 & - & - & 6,18 & - & - & 7,82 & - & - & 0,47 & - & - & $-1,65$ & - & - \\
\hline \multirow{3}{*}{ Biblioteca } & \multicolumn{15}{|c|}{ Total (media de las 3 dimensiones) } \\
\hline & \multicolumn{3}{|c|}{ Mín } & \multicolumn{3}{|c|}{ Obs } & \multicolumn{3}{|c|}{ Des } & \multicolumn{3}{|c|}{ Ade } & \multicolumn{3}{|c|}{ Sup } \\
\hline & $\mathbf{T}$ & $\mathbf{H}$ & M & $\mathbf{T}$ & H & M & $\mathbf{T}$ & H & M & $\mathbf{T}$ & H & M & $\mathbf{T}$ & H & M \\
\hline Salamanca & 5,96 & 5,90 & 5,99 & 6,88 & 6,83 & 6,90 & 8,40 & 8,36 & 8,43 & 0,92 & 0,93 & 0,91 & $-1,53$ & $-1,52$ & $-1,53$ \\
\hline Argentina & 6,50 & 6,47 & 6,52 & 7,49 & 7,40 & 7,54 & 8,43 & 8,30 & 8,52 & 0,98 & 0,94 & 1,01 & $-0,95$ & $-0,90$ & $-0,98$ \\
\hline Chile & 5,90 & - & - & 6,59 & - & - & 7,97 & - & - & 0,69 & - & - & $-1,38$ & - & - \\
\hline
\end{tabular}

Nota: Mín equivale a mínimo, Obs a observado, Des a deseado, Ade a adecuación, Sup a superioridad. H equivale al género hombre, $M$ al género mujer y $\mathrm{T}$ al total. Los valores Mín, Obs y Des se expresan según la escala Likert del 1 (menor satisfacción) al 9 (mayor satisfacción). 
las bibliotecas latinoamericanas se da en la dimensión del valor afectivo del servicio para Chile $(7,11$ frente al 6,31 del espacio y el 6,18 del control de la información) y en la del espacio en Argentina (7,83 frente al 7,78 del valor afectivo y el 6,93 del control de la información). Por tanto, considerando que el nivel observado se refiere a lo que se percibe u observa en la biblioteca durante el uso del servicio, en la biblioteca española la dimensión con un valor observado más alto se da en la del valor afectivo del servicio. Por su lado, en las bibliotecas latinoamericanas el valor observado más bajo se da en la dimensión del control de la información.

En cuanto al valor deseado, en los tres estudios el valor más alto se da en la dimensión del espacio $(8,44$ en Salamanca, 8,57 en Argentina y 8,13 en Chile), y el más bajo en la dimensión del control de la información (8,35 para Salamanca, 8,31 para Argentina y 7,82 para Chile). Por tanto, contando con que el nivel deseado se refiere a lo que a las personas usuarias les gustaría recibir del servicio, en todas las bibliotecas la dimensión con niveles deseados más altos es la del espacio, mientras que la que presenta los niveles más bajos es la del control de la información.

En cuanto a la adecuación, en el caso de Salamanca la dimensión con una adecuación más alta es la del valor afectivo del servicio $(1,20)$, y la más baja se da en la biblioteca como espacio $(0,63)$. Por su lado, en Chile y Argentina, mientras que la dimensión con la adecuación más alta se da también en el valor afectivo del servicio $(1,09$ y 1,18 respectivamente), la más baja en el caso de Chile se da como en el caso español en la del espacio $(0,31)$, y en Argentina en la dimensión del control de la información $(0,69)$. Por tanto, se puede concluir que en el caso español la dimensión que más precisa de mejoras por resultar con la adecuación más baja es la del espacio, y en las bibliotecas latinoamericanas también lo es la del control de la información.

Por último, en cuanto a la superioridad, cabe resaltar que en ninguna dimensión de ningún estudio es positiva. De hecho los resultados más altos se dan en la biblioteca de Argentina con -0,69 en la dimensión del valor afectivo del servicio, muy lejos aún del 0 y de lo positivo. De todo ello se puede concluir que ninguna biblioteca consigue ofrecer un servicio que quede por encima de las expectativas de las personas usuarias en lo referido a alguna de las dimensiones.

\subsection{Diferencias en los resultados considerando la variable de género en Argentina y Salamanca}

En cuanto al valor mínimo, se observa que en Salamanca en el género mujer se da mayor exigencia que en los hombres en las tres dimensiones. Además, el mínimo total media de las tres dimensiones es más alto en el género mujer que en el hombre en dicho estudio $(5,99$ frente a 5,90). Por su lado, en Argentina se da mayor exigencia en el género mujer que en el género hombre en la dimensión del valor afectivo (6,65 frente a 6,51), pero no en la del espacio $(6,75$ en hombres y 6,74 en mujeres) ni en la del control de la información (en este caso, aunque el resultado en la Tabla VI resultó de 6,24 para hombres y mujeres, el tercer decimal hace mayor la exigencia de hombres que en mujeres, 6,244 frente a 6,243 respectivamente). Además, en la biblioteca de Argentina también el mínimo total media de las tres dimensiones es más alto en las mujeres que en los hombres $(6,52$ frente a 6,47). Por tanto, considerando el mínimo total media de las tres dimensiones, tanto en la biblioteca española como en la argentina el nivel de exigencia del género mujer es mayor que en el caso del género hombre. Además, cabe resaltar que en la dimensión del valor afectivo en los dos estudios el nivel de exigencia es mayor en mujeres que en hombres.

En lo referido al valor observado, en el caso español el valor dado por las mujeres es menor que el de los hombres en la dimensión del valor afectivo del servicio (7,20 frente a 7,24 respectivamente) y mayor en las del espacio $(6,64$ frente a 6,45$)$ y el control de la información $(6,73$ frente a 6,62$)$. Además, el valor observado media de las tres dimensiones es más alto en mujeres que en hombres $(6,90$ frente a 6,83). Por su lado, en Argentina, el valor observado dado por las mujeres es mayor que el de los hombres en todas las dimensiones y en el total media de las mismas. Por tanto, aunque el valor observado media de las tres dimensiones es mayor en mujeres que en hombres en la biblioteca española, las mujeres en la biblioteca española perciben niveles menores que los hombres en cuanto al valor afectivo del servicio. Además, las mujeres en la biblioteca de Argentina perciben niveles mayores que los hombres en todas las dimensiones.

En lo concerniente al valor deseado, tanto en la biblioteca española como en la argentina el valor de las mujeres siempre es más alto que el de los hombres por dimensiones y en el total. Por tanto, en el género mujer se da un valor mayor en todos los estudios y dimensiones en relación a los que les gustaría recibir del servicio con respecto al género hombre.

En cuanto a la adecuación, en el caso de Salamanca es más baja en mujeres que en hombres en el valor afectivo $(1,16$ frente a 1,26$)$, y más alta en las dimensiones del espacio $(0,64$ frente a 0,61$)$ y el control de la información $(0,79$ frente a 0,76$)$. Aun con todo, en Salamanca la adecuación media de las tres dimensiones es más baja en mujeres que en hombres (0,91 frente a 0,93). En el caso de Argentina, la adecuación para las mujeres es siempre más alta que la de los hombres en las tres dimensiones y el total. Por tanto, mientras que en el caso de Argentina la adecuación en las mujeres es mayor, en Salamanca sucede que la adecuación es menor para mujeres en la dimensión del valor afectivo del servicio. Por tanto, en la biblioteca española la dimensión del valor afectivo del servicio es la más resentida y con menor adecuación para el género mujer. 
Por último, los resultados para la superioridad en el caso del valor afectivo del servicio son menos negativos en hombres que en mujeres en ambos estudios. En el espacio sucede lo anterior en Argentina $(-0,71$ para hombres y $-0,76$ para mujeres $)$, pero no así en Salamanca, siendo la superioridad menos negativa en el caso de las mujeres (de $-1,91$ y $-1,86$ para hombres y mujeres respectivamente). Por su lado, en el control de la información la superioridad es menos negativa para hombres en Argentina $(-1,27$ frente a $-1,44$ en mujeres), y para mujeres en Salamanca $(-1,63$ frente a $-1,71$ en hombres). Por último, para los resultados totales media de las 3 dimensiones la superioridad es menos negativa en hombres en ambos estudios (en Argentina -0,90 para hombres y $-0,98$ para mujeres y en Salamanca de $-1,52$ para hombres y de $-1,53$ para mujeres).

Por tanto, aunque los resultados de la superioridad son negativos siempre y lejos del 0 , sí que se puede decir que en los dos estudios se está más cerca de superar las expectativas del género hombre en la dimensión del valor afectivo del servicio que las del género mujer. Para Argentina, sucede lo mismo en las dimensiones del espacio y el control de la información, y no así en el caso de Salaman$\mathrm{ca}$, donde en dichas dimensiones se estaría menos lejos de superar las expectativas del género mujer.

\subsection{Análisis estadístico sobre las diferencias por género}

El análisis estadístico se basa en la aplicación de la prueba no paramétrica U de Mann-Whitney aplicada a dos muestras independientes (géneros hombre y mujer) dada la ausencia de normalidad en las variables analizadas. La ausencia de normalidad en las variables analizadas se estudió mediante la prueba de Kolmogorov-Smirnov debido al tamaño de las muestras en los diferentes estudios LibQUAL $+\mathbb{R}$ (siempre mayor que 50). Los resultados de las pruebas U de Mann-Whitney se incluyen en la Tabla VII.

Tal y como se observa, se encontraron diferencias estadísticamente significativas en seis casos del estudio de Argentina y dos en el de Salamanca. En el caso de Argentina, las diferencias en cuanto a género resultaron estadísticamente significativas en los casos del valor observado en la dimensión del valor afectivo del servicio, en el deseado de las dimensiones del valor afectivo, la biblioteca como espacio y el control de la información y en el total media de las tres dimensiones, y en el caso de la superioridad en el control de la información. Por su lado, en el estudio de Salamanca la diferencia resultó significativa en el mínimo y el deseado de la dimensión del espacio. Por tanto, se encontró un caso en que en un indicador propio de la metodología LibQUAL $+\circledR$ la diferencia en cuanto a género resultó estadísticamente significativa: el caso de la superioridad en el control de la información para la biblioteca de Argentina. Los demás casos hallados se refieren a valores mínimos, observados y deseados.
Con todo, el resultado de la diferencia estadísticamente significativa de la superioridad en el control de la información para la biblioteca de Argentina supone un hallazgo relevante con respecto a los estudios realizados sobre diferencias de género en estudios LibQUAL+ $\circledR$ mencionados en la revisión de la literatura, hecho que se analiza en la siguiente sección sobre la discusión de los resultados.

\section{DISCUSIÓN}

Los objetivos de este trabajo se plantearon como respuesta a cuatro preguntas de investigación. La primera de ellas se refería a las diferencias en los resultados por dimensiones entre España y los países latinoamericanos considerando al total de personas usuarias; la segunda y la tercera, a las principales diferencias atendiendo a la variable de género y a si dichas diferencias resultaban estadísticamente significativas; la cuarta y última, a si los resultados hallados en el ámbito concreto de las bibliotecas pueden considerarse reflejo de la realidad social. En cuanto a las tres primeras preguntas se marcaron dos hipótesis: la de que la dimensión mejor valorada en las bibliotecas sería la del control de la información, por la común idea que asocia a las bibliotecas con la información; y la de que los resultados según la variable de género y de acuerdo a la literatura existente revisada no serían estadística y significativamente diferentes entre hombres y mujeres. En lo que sigue, se tratan las tres primeras preguntas de investigación en una discusión y reflexión que equivaldría a la cuarta.

En cuanto a la primera pregunta de investigación y a la primera hipótesis planteada, los resultados de este trabajo permiten concluir que la dimensión mejor valorada de acuerdo al indicador de la adecuación es la del valor afectivo del servicio en todos los estudios, bibliotecas y países contemplados. Por tanto, la dimensión mejor valorada es la del valor afectivo del servicio y no la del control de la información como marcaba la hipótesis. Además, se obtuvo que en el caso español la dimensión que más precisa de mejoras es la del espacio, mientras que en las bibliotecas latinoamericanas también lo era la del control de la información.

En un sentido similar pero esta vez en cuanto a los valores mínimos, en las bibliotecas analizadas los resultados apuntan a que se exige más valor afectivo del servicio o espacio que control de la información. A su vez y en cuanto a los valores observados, los más altos en España y en el caso de Chile se dieron en la dimensión del valor afectivo, mientras que en Argentina la dimensión con observados más altos fue la del espacio. Por su lado, los observados más bajos en España se dieron en el espacio y en los casos de Chile y Argentina el observado más bajo se dio en el control de la información. Por último, en todas las bibliotecas la dimensión con niveles deseados más altos resultó la del espacio y con los más bajos la del control de la información. 
Tabla VII. Resultados para la prueba U de Mann-Whitney - Estadísticos de prueba (variable de agrupación: género)

\begin{tabular}{|c|c|c|c|c|c|}
\hline Dimensión & Estudio & U de Mann-Whitney & W de Wilcoxon & $\mathbf{z}$ & Sig. asintótica (bilateral) \\
\hline \multirow{2}{*}{ AdeTotal } & Argentina & 61488,000 & 99714,000 & $-0,520$ & 0,603 \\
\hline & Salamanca & 176566 & 454451 & $-0,370$ & 0,712 \\
\hline \multirow{2}{*}{ AFAde } & Argentina & 61703,500 & 99929,500 & $-0,443$ & 0,658 \\
\hline & Salamanca & 172511,5 & 450396,5 & $-1,041$ & 0,298 \\
\hline \multirow{2}{*}{ AFDes } & Argentina & 51573,500 & 89799,500 & $-4,251$ & $0,000 * * *$ \\
\hline & Salamanca & 173701 & 289141 & $-0,868$ & 0,385 \\
\hline \multirow{2}{*}{ AFMín } & Argentina & 59598,500 & 97824,500 & $-1,202$ & 0,229 \\
\hline & Salamanca & 172410,5 & 287850,5 & $-1,058$ & 0,290 \\
\hline \multirow{2}{*}{ AFObs } & Argentina & 56858,500 & 95084,500 & $-2,198$ & $0,028 *$ \\
\hline & Salamanca & 172318,5 & 450203,5 & $-1,074$ & 0,283 \\
\hline \multirow{2}{*}{ AFSup } & Argentina & 62859,500 & 101085,500 & $-0,025$ & 0,980 \\
\hline & Salamanca & 169109,5 & 446994,5 & $-1,607$ & 0,108 \\
\hline \multirow{2}{*}{ CIAde } & Argentina & 62800,000 & 101026,000 & $-0,046$ & 0,963 \\
\hline & Salamanca & 177767,5 & 455652,5 & $-0,171$ & 0,864 \\
\hline \multirow{2}{*}{ CIDes } & Argentina & 53836,000 & 92062,000 & $-3,357$ & $0,001 * * *$ \\
\hline & Salamanca & 173599,5 & 289039,5 & $-0,884$ & 0,377 \\
\hline \multirow{2}{*}{ CIMín } & Argentina & 62450,000 & 100676,000 & $-0,172$ & 0,863 \\
\hline & Salamanca & 169812 & 285252 & $-1,489$ & 0,136 \\
\hline \multirow{2}{*}{ CIObs } & Argentina & 61882,500 & 100108,500 & $-0,377$ & 0,706 \\
\hline & Salamanca & 173022 & 288462 & $-0,956$ & 0,339 \\
\hline \multirow{2}{*}{ CISup } & Argentina & 57443,500 & 161639,500 & $-1,981$ & $0,048 *$ \\
\hline & Salamanca & 176550 & 291990 & $-0,372$ & 0,710 \\
\hline \multirow{2}{*}{ DesTotal } & Argentina & 51786,000 & 90012,000 & $-4,052$ & $0,000 * * *$ \\
\hline & Salamanca & 169242,5 & 284682,5 & $-1,593$ & 0,111 \\
\hline \multirow{2}{*}{ ESAde } & Argentina & 61346,000 & 99572,000 & $-0,575$ & 0,565 \\
\hline & Salamanca & 178759,5 & 456644,5 & $-0,007$ & 0,995 \\
\hline \multirow{2}{*}{ ESDes } & Argentina & 55194,000 & 93420,000 & $-3,085$ & $0,002 * *$ \\
\hline & Salamanca & 159786,500 & 275226,500 & $-3,276$ & $0,001 * * *$ \\
\hline \multirow{2}{*}{ ESMín } & Argentina & 62823,500 & 101049,500 & $-0,038$ & 0,970 \\
\hline & Salamanca & 165145,500 & 280585,500 & $-2,264$ & $0,024 *$ \\
\hline \multirow{2}{*}{ ESObs } & Argentina & 59809,500 & 98035,500 & $-1,134$ & 0,257 \\
\hline & Salamanca & 170037,5 & 285477,5 & $-1,451$ & 0,147 \\
\hline \multirow{2}{*}{ ESSup } & Argentina & 61125,500 & 165321,500 & $-0,659$ & 0,510 \\
\hline & Salamanca & 177810 & 455695 & $-0,164$ & 0,870 \\
\hline \multirow{2}{*}{ MinTotal } & Argentina & 61115,500 & 99341,500 & $-0,654$ & 0,513 \\
\hline & Salamanca & 167980 & 283420 & $-1,791$ & 0,073 \\
\hline \multirow{2}{*}{ ObsTotal } & Argentina & 58819,500 & 97045,500 & $-1,482$ & 0,138 \\
\hline & Salamanca & 176565 & 292005 & $-0,370$ & 0,712 \\
\hline \multirow{2}{*}{ SupTotal } & Argentina & 59492,000 & 163688,000 & $-1,240$ & 0,215 \\
\hline & Salamanca & 175435,5 & 453320,5 & $-0,557$ & 0,578 \\
\hline
\end{tabular}

$* \mathrm{p} \leq 0,05 ; * * \mathrm{p} \leq 0,01 ; * * * \mathrm{p} \leq 0,001$.

Nota: AFObs y AFDes equivalen respectivamente al valor observado y el deseado de la dimensión del valor afectivo; ESDes, al deseado de la dimensión de la biblioteca como espacio; CIDes, al deseado del control de la información; CISup, a la superioridad de la dimensión del control de la información; TotalDes, al valor deseado total (media de las 3 dimensiones del estudio); y ESmín al valor mínimo de la dimensión del espacio. 
Con todo, los resultados apuntan a una dirección distinta de la marcada en la hipótesis acerca de que la dimensión mejor valorada y en la que se centrarían los intereses de las personas usuarias sería la del control de la información. En el caso español las personas usuarias están exigiendo mejoras en lo que a espacio se refiere, y en los casos latinoamericanos en lo concerniente al control de la información.

Al respecto y poniendo en relación los resultados del presente trabajo en torno a la primera pregunta de investigación con los vistos en la revisión de la literatura sobre el tema, cabe resaltar que en el estudio llevado a cabo por McCaffrey (2013) en bibliotecas universitarias de Irlanda la dimensión sobre la que las personas usuarias eran más exigentes y sobre la que había mayor descontento fue también la relacionada con los edificios y el espacio. Por su lado, en el estudio de Killick y otros (2014) en bibliotecas de los Países Bajos, la dimensión mejor valorada fue la del valor afectivo del servicio y la peor la del espacio. Igualmente, en el estudio sobre bibliotecas biosanitarias españolas de López-Gijón y otros (2010), el espacio resultó como la dimensión con niveles deseados más altos y las del valor afectivo del servicio y el espacio sobre las que daba una mayor exigencia. Los resultados de los 3 estudios europeos anteriores concuerdan en buena medida con los hallados en este trabajo para la biblioteca española, pues la mayor exigencia de mejoras fue para la dimensión de la biblioteca como espacio y la dimensión mejor valorada la del valor afectivo del servicio.

Por otro lado, en el estudio llevado a cabo en Irán por Asemi y otros (2010), la dimensión sobre la que se percibe menor grado de satisfacción también fue la del espacio, pero la mejor valorada resultó la del control de la información. En cambio, en el estudio también en Irán de Pourahmad y otros (2016), el valor afectivo del servicio resultó con la adecuación más alta seguida del espacio y con la del control de la información en último lugar. Este caso coincide con lo hallado en las bibliotecas latinoamericanas del presente trabajo, donde el control de la información resultó la dimensión con peor valoración. A su vez, eso mismo sucede en el estudio de Rehman (2013) en bibliotecas de Pakistán, donde la dimensión peor valorada fue la del control de la información. Por su lado, en el estudio de Dahan y otros (2016) en bibliotecas de Malasia la dimensión con mayor adecuación resultó la relacionada al personal o valor afectivo, como en las tres bibliotecas analizadas en este trabajo.

De todo ello puede extraerse y plantearse la idea de que en regiones como Europa, las personas usuarias están exigiendo mejoras en lo concerniente al espacio, mientras que en regiones como Latinoamérica, y otras de Asia occidental o Asia del sur, se exige más en relación al control de la información. Desde ahí puede también extraerse la idea de que las exigencias hacia las bibliotecas varían de región a región de acuerdo al desarrollo económico y social del entorno en el que se hallan, resultando que en regiones más avanzadas o desarrolladas como Europa las bibliotecas han dado ya un paso más que en las otras regiones mencionadas, en las cuales se exige control de la información y se sigue identificando a la biblioteca con dicha dimensión. En Europa, en cambio, la biblioteca puede haber pasado ya a ser algo más que solo una colección de documentos. De hecho, son numerosos los trabajos a nivel internacional que plantean a la biblioteca como tercer lugar junto al hogar y al trabajo (Houghton y otros, 2013; Lin y otros, 2015) y por su rol e importancia para la comunidad en la que se hallan (Herrera-Viedma y López-Gijón, 2013). A su vez, recientemente ha surgido una nueva identidad de las bibliotecas en su relación con los makerspaces tanto en bibliotecas públicas (Slatter y Howard, 2013; Leorke y otros, 2018) como en universitarias (Okpala, 2016; Xi y otros, 2017). En ese sentido, las bibliotecas europeas estarían más cerca de estas nuevas identidades y paradigmas que en el caso de otras regiones, idea que se ve fortalecida por los resultados hallados en este trabajo.

Concretando el marco de las bibliotecas universitarias como pertenecientes a la institución educativa de las respectivas universidades, pueden también valorarse los resultados de las universidades a las que pertenecen las bibliotecas incluidas en este estudio en rankings internacionales de investigación. Así, en los últimos años ninguna universidad aparece en el top 500 del ranking ARWU de Shanghai (ARWU, 2020). Tan solo la Universidad de Salamanca aparece en las posiciones 701-800. A su vez, en la última edición del ranking Webometrics del Consejo Superior de Investigaciones Científicas de España, concretamente al ranking referido a la excelencia y que mide el número de publicaciones en el top del $10 \%$ más citadas en 26 disciplinas para los años 2012-2016 obtenidos de Scimago, la Universidad de Salamanca resulta en la posición 667 a nivel mundial y las de Chile y Argentina en las posiciones 844 y 3446 respectivamente (Webometrics, 2020). Cabe resaltar que justo es la Universidad de Salamanca, donde la dimensión de la biblioteca sobre la que más se exige mejoras es la del espacio, la que obtiene mejores resultados con respecto a las de Chile y Argentina en los rankings internacionales mencionados sobre producción científica. Con todo, dicha relación puede servir para presentar la idea de que en contextos más desarrollados tanto social como científicamente hablando, las bibliotecas ya tienen más desarrollada la dimensión del control de la información de acuerdo a las necesidades de las personas usuarias, dando lugar a nuevas y mayores exigencias de mejora en lo referido a la dimensión del espacio. En cambio, en países con menor grado de desarrollo, todavía se exige mejoras en lo relacionado al control de la información. 
En cuanto a las preguntas de investigación segunda y tercera acerca de las diferencias en los resultados por la variable de género, mientras que en la biblioteca de Argentina la adecuación en las mujeres resultó mayor que la de los hombres en todas las dimensiones y el total media de las tres dimensiones, en Salamanca resultó que la adecuación fue menor para mujeres en el valor afectivo del servicio y mayor en el resto de casos.

En ese sentido, considerando el mínimo total media de las tres dimensiones también se vio que el nivel de exigencia de las mujeres resultó mayor que el de los hombres al ser su mínimo más alto tanto en la biblioteca de Salamanca como en la de Argentina. Además, en la dimensión del valor afectivo en ambos estudios el nivel de exigencia al servicio fue mayor en mujeres que en hombres. A su vez, aunque el valor observado media de las tres dimensiones fue mayor en mujeres que en hombres en la biblioteca española, las mujeres dieron niveles menores que los hombres en cuanto al valor afectivo. Por otro lado, las mujeres en la biblioteca de Argentina dieron niveles mayores en el observado con respecto a los hombres en todas las dimensiones. Por último y en cuanto al deseado, en el género mujer se da un valor mayor en ambos estudios y todas las dimensiones en relación a lo que les gustaría recibir del servicio en comparación al de los hombres.

Desde ahí, se analizó si la diferencia según la variable de género resultaba estadísticamente significativa tanto de los valores mínimos, observados y deseados y los indicadores de la adecuación y la superioridad. Los resultados mostraron que existen diferencias significativas entre géneros en el caso de la superioridad del control de la información en Argentina y varios niveles mínimos, observados y deseados en dicho estudio y en el de Salamanca. Por tanto, el hallazgo en la superioridad anterior de Argentina se contrapone a la segunda hipótesis acerca de que los resultados según la variable de género y de acuerdo a la literatura sobre el tema no serían estadísticamente diferentes para los géneros hombre y mujer (Hariri y Afnani, 2008; Cook y Thompson, 2001).

Comparando los resultados hallados en este trabajo con los mencionados al respecto de las preguntas de investigación segunda y tercera en la revisión de la literatura, cabe señalar que en el estudio de Hariri y Afnani (2008) en bibliotecas de Irán la adecuación y la superioridad fueron mayores en mujeres que en hombres en las dimensiones del espacio y el control de la información y menores en la del valor afectivo del servicio. Para la media de las tres dimensiones en dicho estudio, la adecuación y la superioridad resultaron mayores en mujeres que en hombres. Estos resultados difieren en gran medida con los hallados en el presente estudio: por un lado, en Salamanca la adecuación resultó más baja en mujeres que en hombres en el valor afectivo, y más alta en las dimensiones del espacio y el control de la información. Además, la adecuación media en dicha biblioteca resultó más baja en mujeres que en hombres; por otro lado, en el caso de Argentina, la adecuación para las mujeres resultó siempre más alta que la de los hombres en las 3 dimensiones y el total, resultados que coinciden en lo referente al espacio y el control de la información con los del estudio de Irán.

Al respecto de las diferencias de género por dimensiones cabe subrayar el hecho de que las mujeres en la biblioteca española están exigiendo mejoras y muestran menor grado de satisfacción que los hombres en relación al valor afectivo del servicio, hecho que no sucede en la biblioteca Argentina, donde la adecuación siempre fue mayor para mujeres que para hombres. Además, en la biblioteca española el observado fue menor para mujeres que para hombres en el valor afectivo, mientras que en las dimensiones del espacio y el control de la información lo fue para hombres, hecho que apoya la idea de que para las mujeres el valor afectivo del servicio es la dimensión más resentida en la biblioteca española. En ese sentido, para la biblioteca española el mínimo y el deseado fueron siempre mayores en mujeres que en hombres en todas las dimensiones, muestra de que están exigiendo y deseando más al respecto. Con todo, los resultados de este trabajo permiten plantear la cuestión de por qué en España el género mujer manifiesta menor satisfacción y exige más que el género hombre en la dimensión del valor afectivo del servicio, dando pie a una reflexión mayor e interdisciplinar acerca de la situación de la mujer con respecto al hombre en sociedades como la española.

Por otro lado, en el trabajo de Hariri y Afnani (2008) no se hallaron diferencias estadísticamente significativas en cuanto al género. Además, aunque los autores consolidaban la escasa existencia de investigación al respecto, sí que relacionaban sus resultados sobre la falta de diferencias estadísticamente significativas con lo afirmado por Cook y Thompson (2001) acerca de que los resultados en Libqual no serán estadísticamente diferentes en el análisis de la variable de género. A su vez, y aunque en alguna aplicación del modelo Servqual en bibliotecas sí que se hallaron diferencias estadísticamente significativas según dicha variable (Hossain-Shoeb y Zabed-Ahmed, 2009), otros autores como Hiller (2001) refuerzan la idea de la ausencia de diferencias estadísticamente significativas en estudios Libqual. En dicho estudio el autor expone cómo en sus trabajos en la biblioteca de la Universidad de Washington sí se detectaron diferencias significativas en encuestas no basadas en Libqual pero no así en las llevadas a cabo en base a dicha metodología.

En el actual trabajo se detectó un caso en el que sí se dan diferencias estadísticamente significativas en cuanto al género en uno de los indicadores propios de la metodología LibQUAL $+\circledR$, a 
saber, en el caso de la superioridad en la dimensión del control de la información de la biblioteca universitaria de Argentina. Por ello, los resultados hallados fortalecen la idea de Heinrichs y otros (2005) acerca de que los resultados sobre la cuestión de las diferencias estadísticamente significativas respecto al género en estudios de satisfacción de usuarios Libqual no permiten extraer conclusiones firmes, y sobre que el estudio de dichas diferencias es un tema de interés potencial para la investigación.

Con todo, de acuerdo a los resultados hallados podrían establecerse dos paradigmas posibles para las bibliotecas actuales dependiendo del contexto social en el que se hallan y al que prestan sus servicios. Por un lado estaría la biblioteca informativa, paradigma propio de países aun en vías de desarrollo, y que en este artículo se identifican con las zonas de Latinoamérica, Asia occidental y Asia del sur. Por otro lado, estaría la biblioteca socialcreadora, paradigma propio de países o zonas más desarrolladas como Europa, donde ya no solo se espera información de las bibliotecas, que se concibe como algo secundario, sino lo relacionado a espacios para la socialización y la creación.

Aunque la crítica podría calificar la reflexión anterior de eurocentrista al atribuir al desarrollo de países en general y bibliotecas en particular la dirección única más posible y más válida de lo que sucede en Europa, sí puede extraerse la idea de que el desarrollo económico y social de un país tiene su reflejo en la situación de sus bibliotecas. Lo anterior lleva a pensar que cuando los países en vías de desarrollo alcancen su siguiente nivel, también sus bibliotecas llegarán al paradigma de las bibliotecas social-creadoras. Dicho reflejo también se atribuye a las diferencias halladas en este artículo sobre la cuestión de género. En países de Europa, donde la lucha por la superación de las diferencias entre mujeres y hombres está más consolidada (aunque el camino por recorrer pueda ser aún muy largo) y donde se da un mayor desarrollo social con respecto a países de Latinoamérica o Asia occidental y del sur, se halló que en el ámbito concreto de las bibliotecas las mujeres eran más exigentes y mostraban menor grado de satisfacción que los hombres en lo referido al valor afectivo del servicio. Si se parte de la idea de que la biblioteca puede aportar beneficios en el sentido del capital humano, social y cultural (Lázaro-Rodríguez y otros, 2018b), sus servicios habrían de adaptarse y sus modelos de gestión dar prioridad a la superación de las desigualdades en lo concerniente a la cuestión de género.

Con todo, las implicaciones anteriores pueden servir para prever en el momento actual lo que sucederá en el futuro en el ámbito concreto de las bibliotecas, facilitando la anticipación para ir adaptando los servicios que ofrecen a las exigencias del mañana.

\section{CONCLUSIÓN}

Este trabajo puede ser considerado como una mirada actual a los estudios de satisfacción de personas usuarias en bibliotecas con originalidad y base científica. Se ha tratado de alcanzar nuevo conocimiento comparando los resultados de tres estudios llevados a cabo en bibliotecas universitarias de Argentina, Chile y España. Los temas esenciales abordados han sido el de las principales diferencias sobre la valoración de las dimensiones del valor afectivo del servicio, la biblioteca como espacio, y el control de la información o servicios prestados; y el de las principales diferencias atendiendo a la variable de género incluyendo un análisis para comprobar si las diferencias resultaban estadísticamente significativas. Los resultados hallados en el campo concreto de las bibliotecas se pusieron en relación con la realidad social en general. Desde ahí, se llevó a cabo una reflexión en torno a dichos resultados como reflejo de dicha realidad y se definieron dos paradigmas como posibles para las bibliotecas actuales: el de la biblioteca informativa y el de la biblioteca social-creadora: el primero propio de sociedades, países y regiones aun en vías de desarrollo, y el segundo propio de contextos con mayor grado de desarrollo.

Como limitación principal del trabajo puede considerarse que las conclusiones alcanzadas lo fueron a partir de la interpretación de los resultados de tres estudios concretos. Aun con todo, si bien la primera de las hipótesis marcadas tomaba al control de la información como dimensión en la que las personas usuarias pondrían el foco de la mayor adecuación, satisfacción y exigencia por la común idea que identifica a las bibliotecas con la información, los resultados hallados en el estudio permiten situar el foco de la biblioteca española y las europeas en lo que a la dimensión de la biblioteca como espacio se refiere, y el de las latinoamericanas en lo referido al control de la información. Por otro lado, los resultados de este trabajo permiten poner en duda la idea de otros autores que en trabajos anteriores consolidaban que las diferencias por género en estudios de satisfacción no serían estadísticamente significativas. A ello aludía la segunda de las hipótesis marcadas. Pero el hallazgo de la diferencia estadísticamente significativa en la superioridad del control de la información en el estudio de Argentina permite ser considerado como un contraejemplo a la idea de la no diferencia estadísticamente significativa en estudios de satisfacción de personas usuarias en bibliotecas.

A su vez, otras limitaciones del actual trabajo pueden considerarse como futuras líneas a desarrollar en próximas investigaciones. Por ejemplo, los resultados de los estudios Libqual se basan en la percepción de los usuarios reales y no los potenciales. Tal y como se planteó al tratar sobre la información de la población de los estudios en que se basa este trabajo, resultaría interesante plantear estudios que consideren a las personas no usuarias 
en aras a una evaluación más completa. Por otro lado, es importante resaltar que los resultados del presente estudio se analizaron según las dimensiones del valor afectivo, la biblioteca como espacio y el control de la información. Pero la realidad es que cada dimensión responde a un conjunto de ítems particulares que, aunque se recogen en una misma dimensión, también se diferencian entre sí. En ese sentido, pueden plantearse estudios más específicos cuyo nivel de análisis sean los ítems individuales en vez de por dimensiones, analizando también las diferencias en los resultados por género, apoyándose en los comentarios de los campos opcionales aportados por las personas usuarias añadidos en las encuestas y analizando los resultados por tipo de personas usuarias.

La originalidad de este trabajo también se puede entender en relación a las perspectivas de futuro que abre el mismo, y es que tal y como muestran los resultados alcanzados y en base a la literatura revisada, el estudio de las diferencias en cuanto a género representa un campo potencialmente de interés para la investigación en nuestro campo, por la escasa investigación llevada a cabo al respecto y porque las conclusiones extraídas sobre el tema en trabajos anteriores no pueden considerarse como firmes. En ese sentido, este trabajo aporta nuevo conocimiento ante las conclusiones de dichos trabajos. Además, en relación a las principales diferencias por género y las dimensiones contempladas se planteó una cuestión que puede ser abordada en un ejercicio de reflexión interdisciplinar, y es que en la biblioteca española, a diferencia de lo que sucede en las latinoamericanas, resultó que el género mujer presenta una menor satisfacción y mayor exigencia en lo relacionado al valor afectivo del ser-

\section{NOTAS}

1. Lázaro-Rodríguez, P.; López-Gijón, J.; Alonso, S.; Herrera-Viedma, E. (2019): Colección con 3 documentos para los cuestionarios de los estudios Libqual. Datos de investigación. https://figshare.com. https://doi. org/10.6084/m9.figshare.c.4555547.v4. Se trata de una colección en el repositorio figshare de 3 documentos en formato txt que incluyen los cuestionarios de las encuestas llevadas a cabo en las bibliotecas universitarias de Argentina, Chile y Salamanca.

\section{REFERENCIAS}

Ahmed, S. (2017). Service Quality Satisfaction: A Comparative Cross-Sectional Study of Public and Private University Librarians in Pakistan. Libri, 67 (4), 313-325. https://doi.org/10.1515/libri-2017-0011

ARL. (2018). Association of Research Libraries - Libqual+ 2018 Survey. Disponible en: https://www.libqual.org/ documents/LibQual/notebooks/261_6.pdf [Fecha de consulta: 06/06/2019].

ARWU. (2020). ARWU World University Rankings 2018 । Academic Ranking of World Universities [en línea]. Disponible en: http://www.shanghairanking.com/index. html [Fecha de consulta: 01/04/2020]. vicio. Una pregunta que puede servir para futuras investigaciones interdisciplinares es la que se marque como objetivo hallar razones sobre lo anterior, relacionando ese hecho con la situación de la mujer y las diferencias según género en contextos sociales concretos y en un marco global y general.

Con todo, considerando los resultados alcanzados y los métodos empleados, este trabajo puede servir de base para profundizar en el estudio e intentar mejorar la menor satisfacción del género mujer en lo que a valor afectivo de los servicios en bibliotecas se refiere, y como ejemplo para la mejora de los servicios ofrecidos por las bibliotecas a las personas usuarias en el ejercicio que supone su evaluación.

\section{AGRADECIMIENTOS}

El primer autor es beneficiario de una de las ayudas para contratos predoctorales de Formación de Profesorado Universitario del Ministerio de Educación, Cultura y Deporte (Referencia FPU2014/04213). El trabajo está soportado por el Ministerio de Economía y Competitividad (Referencia TIN2016-75850-R).

\section{ACKNOWLEDGEMENTS}

The first signing author is a beneficiary of one of the grants for pre-doctoral contracts from the University Teacher Training Program FPU-2014 of the Ministry of Education, Culture and Sports of Spain (reference number FPU-2014/04213). This work is supported by the Ministry of Economy and Competitiveness of Spain (reference number TIN201675850-R).

2. Lázaro-Rodríguez, P.; López-Gijón, J.; Alonso, S.; Herrera-Viedma, E. (2019): Colección con 3 documentos para los resultados de los estudios Libqual. Datos de investigación. https://figshare.com. https:// doi.org/10.6084/m9.figshare.c.4555553.v4. Se trata de una colección en el repositorio figshare de 3 documentos en formato csv que incluyen los resultados por encuesta e ítems de las encuestas llevadas a cabo en las bibliotecas de Argentina, Chile y Salamanca.

Asemi, A.; Kazempour, Z.; Rizi, H. A. (2010). Using LibQUAL+TM to improve services to libraries: A report on academic libraries of Iran experience. Electronic Library, 28 (4), 568-579. https://doi. org/10.1108/02640471011065382

Buck, S.; Nutefall, J. E.; Bridges, L. M. (2012). "We thought it might encourage participation." Using lottery incentives to improve LibQUAL+TM response rates among students. The Journal of Academic Librarianship, 38 (6), 400-408. https://doi.org/10.1016/j.acalib.2012.07.004

Cabrerizo, F. J.; López-Gijón, J.; Martínez, M. A.; Morente-Molinera, J. A.; Herrera-Viedma, E. (2017). 
A Fuzzy Linguistic Extended LibQUAL+ Model to Assess Service Quality in Academic Libraries. International Journal of Information Technology \& Decision Making, 16 (01), 225-244. https://doi.org/10.1142/ S0219622015500406

Cabrerizo, F. J.; Pérez, I. J.; López-Gijón, J.; Herrera-Viedma, E. (2012). An Extended LibQUAL+ Model Based on Fuzzy Linguistic Information. En: Torra, V.; Narukawa, Y.; López, B.; Villaret, M. (eds.), Modelling Decisions for Artificial Intelligence pp. 90-101. Berlin, Heidelberg: Springer. https://doi.org/10.1007/978-3-642-34620-0_10

Collins, E.; Stone, G. (2014). Understanding Patterns of Library Use Among Undergraduate Students from Different Disciplines. Evidence Based Library and Information Practice, 9 (3), 51-67. https://doi. org/10.18438/B8930K

Cook, C.; Heath, F. (2001). The ARL "LibQUAL+" Pilot Project. Journal of Library Administration, 35 (4), 4753. https://doi.org/10.1300/J111v35n04_10

Cook, C.; Heath, F.; Thompson, B. (2001). Libqual+: One Instrument in the New Measures Toolbox. Journal of Library Administration, 35 (4), 41-46. https://doi. org/10.1300/J111v35n04_09

Cook, C.; Heath, F.; Thompson, B.; Kyrillidou, M.; Roebuck, G.; Yeager, A. (2014). European College and University Libraries. Disponible en: https://www. libqual.org/documents/LibQual/publications/2014/ Euro_2014.pdf [Fecha de consulta: 06/06/2019].

Cook, C.; Thompson, B. (2001). Psychometric Properties of Scores from the Web-Based LibQUAL+ Study of Perceptions of Library Service Quality. Library Trends, 49 (4), 585-604.

Cook, C.; Thompson, B.; Heath, F.; Thompson, R. (2001). LibQUAL+: Service Quality Assessment in Research Libraries. IFLA Journal, 27 (4), 264-268. https://doi. org/10.1177/034003520102700410

Dahan, S. M.; Taib, M. Y.; Zainudin, N. M.; Ismail, F. (2016). Surveying Users' Perception of Academic Library Services Quality: A Case Study in Universiti Malaysia Pahang (UMP) Library. The Journal of Academic Librarianship, 42 (1), 38-43. https://doi. org/10.1016/j.acalib.2015.10.006

Delgado-López-Cózar, E. (2004). La investigación por encuesta en la biblioteconomía y documentación española: Análisis de las encuestas publicadas en revistas y congresos de la especialidad entre 1976 y 1997. En: Ríos-Hilario, A.B.; Frías-Montoya, J.A. (coords), Metodologías de investigación en Información y Documentación, 2004, pp. 71-136. Salamanca: Ediciones Universidad de Salamanca.

Fernández-Ardèvol, M.; Ferran-Ferrer, N.; Nieto-Arroyo, J.; Fenoll, C. (2018). The public library as seen by the non-users. El Profesional de La Información, 27 (3), 659-670. https://doi.org/10.3145/epi.2018.may.19

Fushimi, M. (2011). Evaluación de bibliotecas universitarias: una propuesta desde las perspectivas objetiva y subjetiva. Buenos Aires: Alfagrama.
Gimeno-Perelló, J. (2008). Evaluación de la calidad en bibliotecas: compromiso con lo público. Buenos Aires: Alfagrama.

Hariri, N.; Afnani, F. (2008). LibQUAL+ (TM) in Iran: a subgroup analysis by gender. Performance Measurement and Metrics, 9 (2), 80-93. http://dx.doi. org/10.1108/14678040810906790

Heinrichs, J. H.; Sharkey, T.; Lim, J.-S. (2005). Relative Influence of the LibQUAL+TM Dimensions on Satisfaction: A Subgroup Analysis. College \& Research Libraries, 66 (3), 248-265. https://doi.org/10.5860/crl.66.3.248

Herrera-Viedma, E.; López-Gijón, J. (2013). Libraries' Social Role in the Information Age. Science, 339 (6126), 1382-1382. https://doi.org/10.1126/ science.339.6126.1382-a

Hiller, S. (2001). Assessing user needs, satisfaction and library performance at the University of Washington Libraries. Library Trends, 49 (4), 605-625.

Hossain-Shoeb, Z. (2010). Perceptions of Library Services by Gender - a Study in Bangladesh. Libri, 60 (4), 331338. https://doi.org/10.1515/libr.2010.028

Hossain-Shoeb, Z.; Zabed-Ahmed, S. M. (2009). Individual differences in service quality assessment: A study of a private university library system in Bangladesh. Performance Measurement and Metrics, 10 (3), 193211. https://doi.org/10.1108/14678040911014194

Houghton, K.; Foth, M.; Miller, E. (2013). The continuing relevance of the library as a third place for users and non-users of IT: the case of Canada Bay. The Australian Library Journal, 62 (1), p. 27-39. https://doi.org/10.1 080/00049670.2013.771764

Killick, S.; Van-Weerden, A.; Van-Weerden, F. (2014). Using LibQUAL+ ${ }^{\circledR}$ to identify commonalities in customer satisfaction: the secret to success? Performance Measurement and Metrics, 15 (1/2), 23-31. https://doi. org/10.1108/PMM-04-2014-0012

Lázaro-Rodríguez, P.; López-Gijón, J.; Alonso, S.; Martínez-Sánchez: M-Á.; Herrera-Viedma, E. (2018a). Secaba-Rank, herramienta online para analizar y evaluar bibliotecas. El profesional de la información, 27 (2), 278-288. https://doi.org/10.3145/epi.2018.mar.06

Lázaro-Rodríguez, P.; López-Gijón, J.; Herrera-Viedma, E. (2018b). Visibilidad de las bibliotecas públicas y la lectura en medios de comunicación españoles frente a otros hechos de la cultura y relación con su uso: medidas para su mayor promoción y difusión. BiD: textos universitaris de biblioteconomia i documentació, 40. http://dx.doi.org/10.1344/BiD2018.40.7

Lázaro-Rodríguez, P.; López-Gijón, J.; Herrera-Viedma, E. (2019). Secaba-Rank a examen: validación de su metodología sobre eficiencia en bibliotecas versus la técnica Data Envelopment Analysis (DEA) y el método Finlandia. El profesional de la información, 28 (3). https://doi.org/10.3145/epi.2019.may.16

Lázaro-Rodríguez, P.; Vakkari, P. (2018). Modelizando el uso de las bibliotecas públicas a través de sus caracte- 
rísticas: estudio comparativo entre España y Finlandia para los préstamos y las visitas. Revista española de documentación científica, 41 (4), e216. https://doi. org/10.3989/redc.2018.4.1544

Leorke, D.; Wyatt, D.; McQuire, S. (2018). "More than just a library": Public libraries in the 'smart city'. City, Culture and Society, 15, 37-44. https://doi. org/10.1016/j.ccs.2018.05.002

LibQUAL+ß. (2020). LibQUAL+® Publications [en línea]. Disponible en: https://www.libqual.org/publications [Fecha de consulta: 01/04/2020].

Lin, H.; Pang, N.; Luyt, B. (2015). Is the library a third place for young people?. Journal of librarianship and information science, 47 (2), 145-155. https://doi. org/10.1177/0961000614532303

López-Gijón, J.; Ávila-Fernández, B.; Pérez-Gálvez, I.J.; Herrera-Viedma, E. (2010). La calidad en las bibliotecas universitarias biomédicas según sus usuarios. El Profesional de la Información, 19 (3), 255-259. https://doi.org/10.3145/epi.2010.may.05

Lor, P. J. (2008). Critical reflections on international librarianship. Mousaion, 25 (1), 1-15. Disponible en: http://hdl.handle.net/2263/9911

Lor, P. J. (2017). International and Comparative Librarianship. En: McDonald, J.D.; Levine-Clark, M. (eds.), Encyclopedia of Library and Information Sciences, Boca Raton: CRC Press. https://doi. org/10.1515/9783110267990

Maidul-Islam, M. D; Zabed-Ahmed, S. M. (2011). Measuring Dhaka University students' perceptions of ease-of-use and their satisfaction with University Library's online public access catalogue. Performance Measurement and Metrics, 12 (3), 142-156. https:// doi.org/10.1108/14678041111196631

McCaffrey, C. (2013). LibQUAL in Ireland: Performance Assessment and Service Improvement in Irish University Libraries. Journal of Academic Librarianship, 39 (4), 347-350. https://doi.org/10.1016/j.acalib.2012.11.036

MECD. (2020). Bibliotecas públicas españolas en cifras [en línea]. Disponible en: http://www.mecd.gob.es/ cultura-mecd/areas-cultura/bibliotecas/mc/ebp/portada.html [Fecha de consulta: 01/04/2020].

Morales, M.; Ladhari, R.; Reynoso, J.; Toro, R.; Sepulveda, C. (2011). Factor structure and psychometric properties of a Spanish version of LibQUAL ${ }^{\mathrm{TM}}$. Performance Measurement and Metrics, 12 (1), 23-37. https://doi. org/10.1108/14678041111124270

Natesan, P.; Aerts, X. (2016). Can library users distinguish between minimum, perceived, and desired levels of service quality? Validating LibQUAL $+\circledR$ using multitrait multimethod analysis. Library \& Information Science Research, 38 (1), 30-38. https://doi.org/10.1016/j. lisr.2016.01.004

Nunnally, J. C. (1978). Psychometric theory. New York: McGraw-Hill.
Okpala, H. N. (2016). Making a makerspace case for academic libraries in Nigeria. New Library World, 117 (9-10), 568-586. https://doi.org/10.1108/NLW-05-2016-0038

Oomes, M.; Audunson, R.; Vakkari, P.; Aabø, S.; Huysmans, F. (2014). Perceived outcomes of public libraries in Finland, Norway and the Netherlands. Journal of Documentation, 70 (5), 927-944. https:// doi.org/10.1108/JD-06-2013-0072

Parasuraman, A.; Zeithaml, V. A.; Berry, L. L. (1988). Servqual: A Multiple-Item Scale For Measuring Consumer Perceptions of service quality. Journal of Retailing, 64 (1), 12-40.

Pourahmad, A. A.; Neshat, M.; Hasani, M. R. (2016). Using LibQUAL Model for Improving the Level of Students' Satisfaction from Quality of Services in Academic Libraries: A Case Study in North Khorasan Province, Iran. Journal of Information \& Knowledge Management, 15 (1), 1650011. https://doi.org/10.1142/S0219649216500118

Rehman, S. U. (2013). Service Quality Assessment in University Libraries of Pakistan. Canadian Journal of Information and Library Science-Revue Canadienne des Sciences de L'Information et de Bibliotheconomie, 37 (1), 59-80. https://doi.org/10.1353/ils.2013.0001

Rey-Martín, C. (1999). La aplicación de los estudios de satisfacción de usuarios en la biblioteca universitaria: el caso de las universidades catalanas. BiD: textos universitaris de biblioteconomia i documentació, 3. http://bid.ub.edu/03rey2.htm

Sin, S.-C. J.; Huysmans, F.; Vakkari, P.; Aabø, S.; Audunson, R.; Oomes, M.; Kwon, N. (2016). Patterns of perceived public library outcomes in five countries. Journal of Documentation, 72 (2), 342-361. https:// doi.org/10.1108/JD-08-2015-0103

Slatter, D.; Howard, Z. (2013). A place to make, hack, and learn: makerspaces in Australian public libraries. The Australian Library Journal, 62 (4), 272-284. https:// doi.org/10.1080/00049670.2013.853335

Thompson, B.; Cook, C. (2002). Stability of the Reliability of LibQual $+{ }^{\mathrm{TM}}$ Scores a Reliability Generalization Meta-Analysis Study. Educational and Psychological Measurement, 62 (4), 735-743. https://doi.org/10.11 $77 / 0013164402062004013$

Thompson, B.; Cook, C.; Kyrillidou, M. (2005). Concurrent Validity of LibQUAL+ ${ }^{\mathrm{TM}}$ Scores: What Do LibQUAL+ ${ }^{\mathrm{TM}}$ Scores Measure? The Journal of Academic Librarianship, 31 (6), 517-522. https://doi.org/10.1016/j.acalib. 2005.08.002

Toledo-Sánchez, M. C.; Maldonado-Radillo, S. E.; LópezTorres, V. G. (2012). Propiedades psicométricas de una adaptación de LibQUAL+. Un estudio en bibliotecas de universidades mexicanas. Biblios: Revista de Bibliotecología y Ciencias de la Información, 49, 1-13. https:// doi.org/10.5195/biblios.2012.72

Universidad de Salamanca. (2020). Observatorio de la Calidad y el Rendimiento Académico [En línea]. Disponible en: http://indicadores.usal.es/portal/ [Fecha de consulta: 01/04/2020]. 
Voorbij, H. (2012). The use of LibQUAL+ by European research libraries. Performance Measurement and Metrics, 13 (3), 154-168. https://doi. org/10.1108/14678041211284704

Webometrics. (2020). Ranking Web of Universities [en línea]. Disponible en: http://www.webometrics.info/en [Fecha de consulta: 01/04/2020].
Xi, O.; Wu, W.; Zhang, Y. (2017). Makerspace Activities in China. Science and Technology Libraries, 36 (4), 425433. https://doi.org/10.1080/0194262X.2017.1392920

Yesmin, S.; Zabed-Ahmed, S. M. (2016). Preference of Bangladesh university students for searching the library catalogue: OPAC or discovery tool? The Electronic Library, 34 (4), 683-695. https://doi.org/10.1108/EL-07-2015-0139 